\title{
Introduction to distributive categories
}

\author{
J. R. B. COCKETT
}

Department of Computer Science, University of Calgary

Received 1 March 1991; revised 24 September 1992

Distributive category theory is the study of categories with two monoidal structures, one of which "distributes" over the other in some manner. When these are the product and coproduct, this distribution is taken to be the law

$$
(A \times B)+(A \times C) \simeq A \times(B+C),
$$

which asserts that the obvious canonical map has an inverse. A distributive category is here taken to mean a category with finite products and binary coproducts such that this law is satisfied.

In any distributive category the coproduct of the final object with itself, $1+1$, forms a boolean algebra. Thus, maps into $1+1$ provide a boolean logic: if each such map recognizes a unique subobject, the category is a recognizable distributive category. If, furthermore, the category is such that these recognizers classify detachable subobjects (coproduct embeddings), it is an extensive distributive category.

Extensive distributive categories can be approached in various ways. For example, recognizable distributive categories, in which coproducts are disjoint or all preinitials are isomorphic, are extensive. Also, a category $\mathbf{X}$ having finite products and binary coproducts satisfying the slice equation $\mathbf{X} \times \mathbf{X} \simeq \mathbf{X} / 1+1$ (due to Schanuel and Lawvere) is extensive. This paper describes a series of embedding theorems. Any distributive category has a full faithful embedding into a recognizable distributive category. Any recognizable distributive category can be "solidified" faithfully to produce an extensive distributive category. Any extensive distributive category can be embedded into a topos.

A peculiar source of extensive distributive categories is the coproduct completion of categories with familial finite products. In particular, this includes the coproduct completion of cartesian categories, which is serendipitously, therefore, also the distributive completion. Familial distributive categories can be characterized as distributive categories for which every object has a finite decomposition into indecomposables.

\section{Introduction}

The purpose of this paper is to present the results obtained during the first three months of 1990 in distributive category theory at the Sydney Category Seminar (SCS) and the Categories in Computer Science Seminar (CICS) held at the University of Sydney and Macquarie University. The nomenclature has evolved since those original discussions and I have taken the liberty of bringing the exposition up to date in that respect. Although many proofs have been omitted, the intention is to leave sufficient detail so that proofs can be reconstructed when the results are not obvious from the development. 
Distributive categories occur (it seems) all over mathematics. However, a primary motivation for this development was the potential computer science applications. It had been realized for some time that distributive categories are the correct formal setting for studying acyclic programs. In particular, the control aspects of programs, which have proved to be difficult to model equationally, are modeled precisely by the coproduct (sum or disjoint union) of distributive categories. Furthermore, distributive categories (and their analogues in finite sum sketches) provide a powerful language for abstract data structure specification.

The coproduct is a fundamental component of almost all complex data structures, yet it has also, all too often, been absent from specification methodologies. The reason for this can probably be traced to the negative results on the existence of initial algebras for distributive (or finite sum) theories. Initial algebras provide a comfortable source of models: their absence was viewed as a cause for concern. However, even for equational theories the correct formal tool is the generic model, and distributive theories do have generic models. Generic models do not live in Set: they are models that sit in formally constructed environments. In this respect, generic models are examples of the "term models" that are becoming more popular in semantics.

It is tempting to think that category theory simply provides nice theoretical models of computational settings. However, there is a growing realization that category theory is also an important medium for computation, and that a constructive categorical setting can be implemented in much the same way as the $\lambda$-calculus can be implemented. Distributive categories stand at a crossroads, as on the one hand they can have a direct computational reality, and on the other they can embody many of the elementary geometric intuitions that underlie mathematical thought. The relationship between geometric intuition and computation is there to be exploited: to enrich, facilitate, and elucidate software implementation.

\section{Basic definitions and overview}

There are two basic types of categories central to (cartesian) distributive category theory:

Distributive categories: terminal object, binary products (i.e. finite products) and binary coproducts, where the binary products "distribute" over coproducts:

$$
(A \times B)+(A \times C) \equiv A \times(B+C) .
$$

This isomorphism is obtained by requiring that the canonical map

$$
\left\langle i \times b_{0} \mid i \times b_{1}\right\rangle:(A \times B)+(A \times C) \longrightarrow A \times(B+C)
$$

be an isomorphism. This is the distributive property.

Extensive (distributive) categories: Categories with finite products, binary coproducts, and pullbacks along coprojections such that the diagram in Figure 1 holds, where (1) and (2) are pullbacks if and only if the top row, $(Z, x, y)$, is a coproduct. This is the extensive property.

It is, of course, the case that every extensive (distributive) category satisfies the distributive property. 


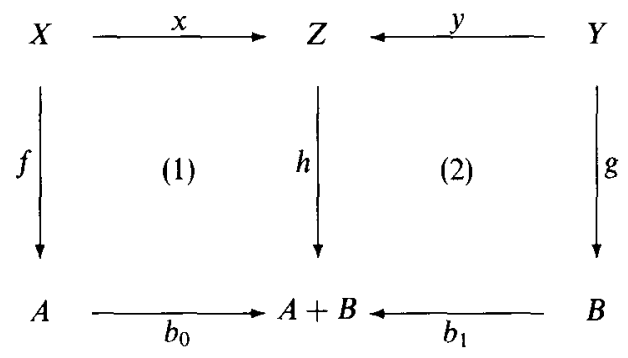

Fig. 1.

Between these extremes lie a number of different variations obtained by demanding specific "local" properties. Recognizable distributive categories are required to have the slice over $1+1$ distributive. This implies that every slice over a sum of final objects (e.g. $\mathrm{n}=1+1+\ldots+1)$ is distributive, giving a fibration over finite sets. The exact relationship between recognizable and extensive distributive will be a central issue in the paper.

I do not demand that distributive categories have an initial object. An initial object, however, can always be added. The gain in dropping this requirement is that some natural examples, which definitely lack initial objects, are then covered.

Demanding that the extensive property holds does affect the structure of the category significantly. The extensive property in the form of a slice condition was introduced by Steve Schanuel at the Sydney category seminar in 1988. Extensive distributive categories have pullbacks over detachable subobjects (essentially coprojections), an initial object, and disjoint coproducts. Unlike distributive categories, they always have a full faithful embedding into a topos.

Finitely complete extensive categories were the categories investigated by Steve Schanuel and Bill Lawvere. They called them distributive categories: consequently they are often referred to as Schanuel/Lawvere distributive categories. These categories seem to crop up again and again in various disparate fields of mathematics.

The diagram in Figure 2 relates the 2-categories involved. Each arrow represents a psuedo 2-adjunction. The horizontal arrows have their units full and faithful functors, while the vertical arrows have only faithful units. The process of passing from the distributive to the extensive property is called solidification. The process, in some sense, represents the passage from computational settings to geometric settings.

Dist is the 2-category of distributive categories with distributive functors, that is functors that preserve finite products and binary coproducts. Dist rec $_{\text {is }}$ ise 2-category of recognizable distributive categories with functors that are not only distributive but preserve recognition. Dist loc $_{\text {is }}$ the 2-category of locally distributive categories with distributive functors preserving recognition. EDist is the 2-category of extensive distributive categories with distributive functors. LEDist is the 2-category of Schanuel/Lawvere distributive categories with distributive functors. Finally Top is the 2-category of topoi.

Of particular interest is the problem of generating free distributive categories from various starting points. Bob Walters, with Shu-Hao Sun, studied this problem starting from: 


$$
A \times(B+C) \simeq(A \times B)+(A \times C)
$$

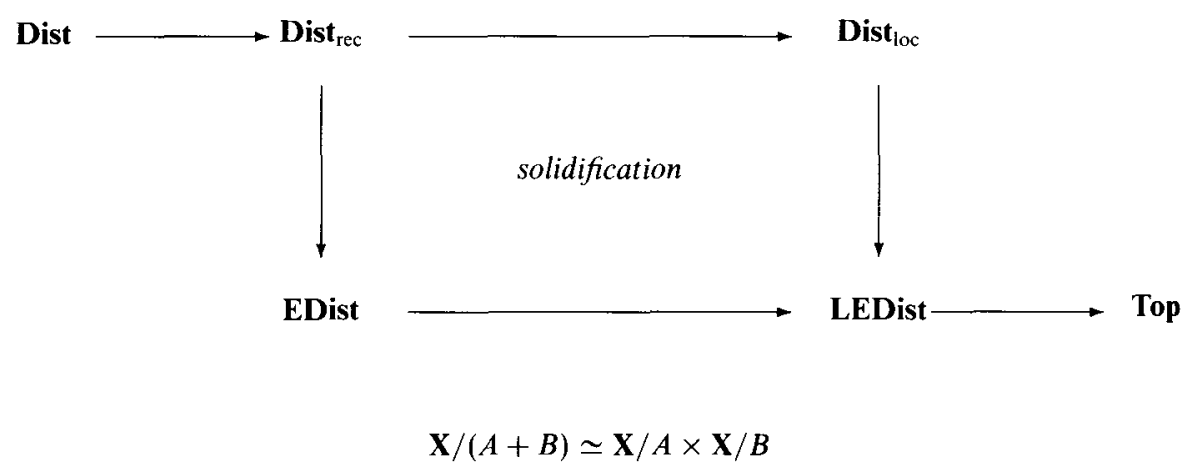

Fig. 2.

- an arbitrary category,

- a category with products,

- a distributive graph or sketch.

Steve Schanuel also studied the first of these problems and gave a "one step" construction, as opposed to the two step construction (first freely add products, then freely add coproducts) advocated by Bob Walters and Shu-Hao Sun. It turns out that using the "family" construction to freely add coproducts to a cartesian category automatically produces a distributive category (observed by Shu-Hao Sun). Indeed, it is the free distributive category on the cartesian category. Rather different is the construction from a distributive graph: it is necessary to use essentially algebraic techniques to generate the initial free distributive category on the given data. One is assured, from general principles, that such exists, because initial models for all finite limit sketches exist (designated products, coproducts, terminal, and initial are implied in these constructions, and some extra argumentation is needed to remove such assumptions).

Free distributive categories in this sense have also been approached from the point of view of their term logic (Cockett 1989). This gives yet another perspective. In particular, there are techniques for "reducing" the terms of this logic, which process corresponds to optimizing the corresponding acyclic code.

A familial distributive category is a distributive category for which every object has a finite (possibly empty) decomposition as a finite coproduct of indecomposables. These categories are equivalent to the coproduct completion of the full subcategory of their indecomposables. The full subcategories $\mathbf{A}$ that are determined by the indecomposables of familial distributive categories, are precisely categories that have familial products. The coproduct completion, of course, is given by the "family" construction, which I shall write as $\mathscr{S} / / \mathbf{A}$ following Ross Street. The category $\boldsymbol{A}$ has familial products if and only if $\mathscr{S} / / \mathbf{A}$ has products. As this category embeds in the distributive category Sets ${ }^{\mathbf{A} p}$ preserving 
limits and colimits, it must itself be distributive. Thus, a distributive category is a familial distributive category precisely if it is equivalent to $\mathscr{S} / / \mathbf{A}$ for some $\mathbf{A}$ : whence the name.

Familial distributive categories are always extensive. They provide a good source of both examples and counter-examples. $G$-Set S $_{f}$, that is the category of actions of a group $G$ in the category of finite sets $\mathbf{S e t}_{f}$, provides a classic example of a familial distributive category. In fact, $G$-Set f $_{f}$ also has each object decidable. That is each object has a decomposition of its product as a diagonal and an off-diagonal. Extensive distributive categories with this property are finitely complete, so they are Schanuel/Lawvere distributive. In fact, familial distributive categories with decidable objects begin to look very like $G$-Set f $_{f}$ and have a number of interesting properties.

The distributive completion of a cartesian category is also an example of a familial distributive category. In fact, the familial distributive category resulting from the coproduct completion of a cartesian category can be characterized in various ways: as having indecomposables whose products are indecomposable or as having the component counting functor (which gives the familial fibration) distributive.

Familial distributive categories have (relatively) simple Burnside rigs (rings in which additive inverses are not assumed), which can always be faithfully extended to Burnside rings. This is another reason why they are of interest. In particular, the various rigs (and, of course, their ring counterparts) of G-Set ${ }_{f}$ have been extensively studied.

\section{Distributive categories}

A distributive category is a category with finite products (including a final object), and with binary coproducts such that the product distributes over the coproduct. Explicitly, this means that the map

$$
\left\langle b_{0} \times i_{A} \mid b_{1} \times i_{A}\right\rangle: B_{1} \times A+B_{2} \times A \longrightarrow\left(B_{1}+B_{2}\right) \times A
$$

is an isomorphism, whose inverse is denoted

$$
d_{0}:\left(B_{1}+B_{2}\right) \times A \longrightarrow B_{1} \times A+B_{2} \times A .
$$

It follows that

$$
\left\langle i_{A} \times b_{0} \mid i_{A} \times b_{1}\right\rangle: A \times B_{1}+A \times B_{2} \longrightarrow A \times\left(B_{1}+B_{2}\right)
$$

is invertible, with inverse denoted by

$$
d_{1}: A \times\left(B_{1}+B_{2}\right) \longrightarrow A \times B_{1}+A \times B_{2} .
$$

Note that a distributive category need not have an initial object. An interesting way in which a distributive category can arise is as the idempotent completion (otherwise known as the Cauchy or Karoubi completion) of a cartesian category. In particular, the idempotent completions of Bool, the theory of Boolean algebras, and Prim the formal theory of primitive recursive functions are distributive (and lack initial objects). There are general conditions that cause the idempotent completion of a cartesian category to be distributive (Cockett 1991). 


\subsection{Basic results}

A fundamental observation concerning the coproduct in any distributive category is given by the following lemma.

Lemma 3.1. In any distributive category, coproduct coprojections are monic.

Proof. Suppose $b_{0}: A \longrightarrow A+B$ and $f, g: C \longrightarrow A$ with $f . b_{0}=g . b_{0}$, then certainly

$$
\left\langle p_{0} . f . b_{0}, i\right\rangle=\left\langle p_{0} . g . b_{0}, i\right\rangle: C \times A \longrightarrow(A+B) \times(C \times A),
$$

but then

$$
\left\langle p_{0} . f \cdot b_{0}, i\right\rangle \cdot d_{0}=\left\langle p_{0} \cdot g \cdot b_{0}, i\right\rangle \cdot d_{0}: C \times A \longrightarrow A \times(C \times A)+B \times(C \times A),
$$

where

$$
\left\langle p_{0} . f, i\right\rangle \cdot b_{0}=\left\langle p_{0} . f . b_{0}, i\right\rangle \cdot d_{0}=\left\langle p_{0} . g . b_{0}, i\right\rangle \cdot d_{0}=\left\langle p_{0} . g, i\right\rangle \cdot b_{0}
$$

However, the embedding

$$
b_{0}: A \times(C \times A) \longrightarrow A \times(C \times A)+B \times(C \times A)
$$

is certainly monic, as it is a section of

$$
\left\langle i \mid p_{1} \cdot\left\langle p_{1}, i\right\rangle\right\rangle: A \times(C \times A)+B \times(C \times A) \longrightarrow A \times(C \times A) .
$$

This means that $\left\langle p_{0} . f, i\right\rangle=\left\langle p_{0} . g, i\right\rangle$, so $p_{0} . f=p_{0} . g$, and, as this projection is a retract (with section $\langle i, f\rangle)$, it follows that $f=g$. Thus, the original $b_{0}$ was monic.

This legitimizes the term embedding for coprojection. ${ }^{\dagger}$

A distributive category does not require an initial object to be present, nor must distributive functors preserve such an object. Despite this, objects that are nearly initial play a key role.

A preinitial object is an object with at most one map to each given object. A preinitial is strict if every object with a map to it is a preinitial object.

A preinitial need not be an initial object, as it need not have a map to every object in the category. If the category has an initial object, the preinitial objects are "epimorphs" of it, so might be regarded as "cotruth" values. Notice that an initial object is strict precisely when every map to it is an isomorphism. Also notice that a strict preinitial is a subobject of every object to which it has a map.

Lemma 3.2. In a category with coproducts, the following are equivalent:

(i) $P$ is preinitial,

(ii) whenever there is a map $p: P \longrightarrow X$, the embedding $b_{0}: X \longrightarrow X+P$ is an isomorphism,

(iii) $b_{0}: P \longrightarrow P+P$ is an isomorphism,

$\dagger$ This fact has often been overlooked: for example the definition of "universal disjoint coproducts", occurring everywhere, but probably originating in SGA 4 (Grothendiek and Verdier 1972), leads one to assume that the requirement that the embeddings be monic is an independent requirement: this is not the case. In fact, as shall be seen, even the requirement of disjointness is not totally independent, as universal coproducts are nearly disjoint. 


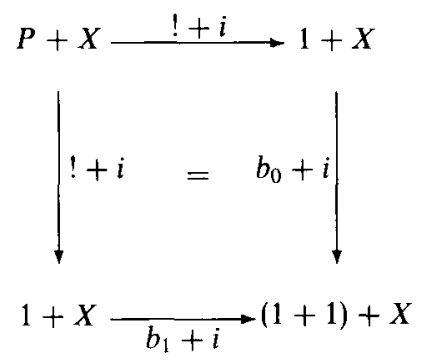

Fig. 3.

(iv) the codiagonal map $\langle i \mid i\rangle: P+P \longrightarrow P$ is an isomorphism,

(v) $b_{0}=b_{1}: P \longrightarrow P+P$.

The proof of these equivalences is straightforward. In a distributive category even more is true:

Proposition 3.3. In any distributive category the following are equivalent:

(i) $P$ is preinitial,

(ii) there is an object $X$ such that $b_{0}: X \longrightarrow X+P$ is an isomorphism,

(iii) $! . b_{0}=! . b_{1}: P \longrightarrow 1+1$.

\section{Proof.}

(i) $\Rightarrow$ (ii)

$b_{0}: P \longrightarrow P+P$ is an isomorphism.

(ii) $\Rightarrow$ (iii)

The square in Figure 3 commutes, as preceding the maps by $b_{1}$ reduces them to the identity. But

$$
! . b_{0} \cdot b_{0}=b_{0} \cdot\langle!+i\rangle \cdot\left\langle b_{0}+i\right\rangle=b_{0} \cdot\langle!+i\rangle \cdot\left\langle b_{1}+i\right\rangle=! . b_{1} \cdot b_{0} .
$$

Now, using the fact that $b_{0}$ is monic (notice that it is not necessary to use the above result for this, as it is a section of $\left.\left\langle i \mid ! . b_{0}\right\rangle\right)$ gives $! . b_{0}=! . b_{1}$.

(iii) $\Rightarrow$ (i)

If $! . b_{0}=! . b_{1}: P \longrightarrow 1+1$, then the square in Figure 4 commutes. Notice that distribution over $1+1$ has been used to obtain this diagram. This means $p_{0} . b_{0}=$ $p_{0} b_{1}: P \times P \longrightarrow P+P$. However, this $p_{0}$ is split by the diagonal and so is a retract. This shows that the embeddings are equal and that $b_{0}=b_{1}: P \longrightarrow P+P$, which shows that $P$ is preinitial.

The proof of the last equivalence (iii) $\Rightarrow$ (i) is due to Mike Johnson, and was a significant improvement on the non-elementary proof of these facts that I gave. In particular, notice that only this step requires distributivity, and, in fact, only the distributivity of the product over $1+1$. These various equivalent descriptions of preinitial allow the observations contained in the following corollary. 


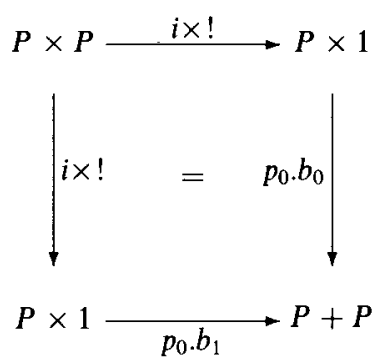

Fig. 4.

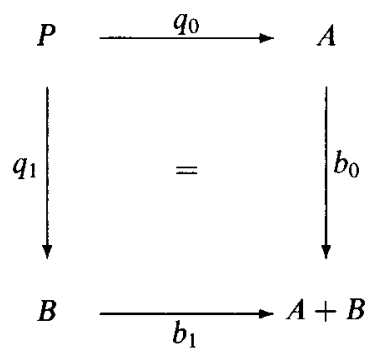

Fig. 5 .

Corollary 3.4. In a distributive category

(i) preinitials are strict,

(ii) if an initial object exists, it is necessarily a strict initial object, ${ }^{\dagger}$

(iii) if $P$ is a preinitial and $X$ is any object, then $P \times X$ is preinitial,

(iv) if any preinitial exists, every object has a preinitial subobject,

(v) if the square in Figure 5 commutes, then $P$ is preinitial.

\subsection{Decomposition to preinitials and solid objects}

A prelattice is a preorder with products and coproducts. A prelattice is unbounded if it lacks a top and bottom.

Lemma 3.5. The full subcategory $\operatorname{Pr}(\mathbf{X})$ of preinitials of a distributive category is a, possibly unbounded, distributive prelattice.

A final preinitial is a preinitial object 0 such that every preinitial has a (necessarily unique) map to it. A distributive category is quasi-solid when it has a final preinitial, and solid when this final preinitial is initial.

Observe that if a final preinitial is initial, every preinitial is isomorphic to it. As every object $X$ in a quasi-solid distributive category contains a preinitial, namely $p_{0}: X \times 0 \longrightarrow$ $X$, a quasi-solid category in which all preinitials are isomorphic must be solid.

$\dagger$ Bob Walters originally gave the definition of a distributive category as having finite products and coproducts such that not only was the distributive law satisfied but also the law $X \times 0 \simeq 0$. In retrospect, this is already implied. 


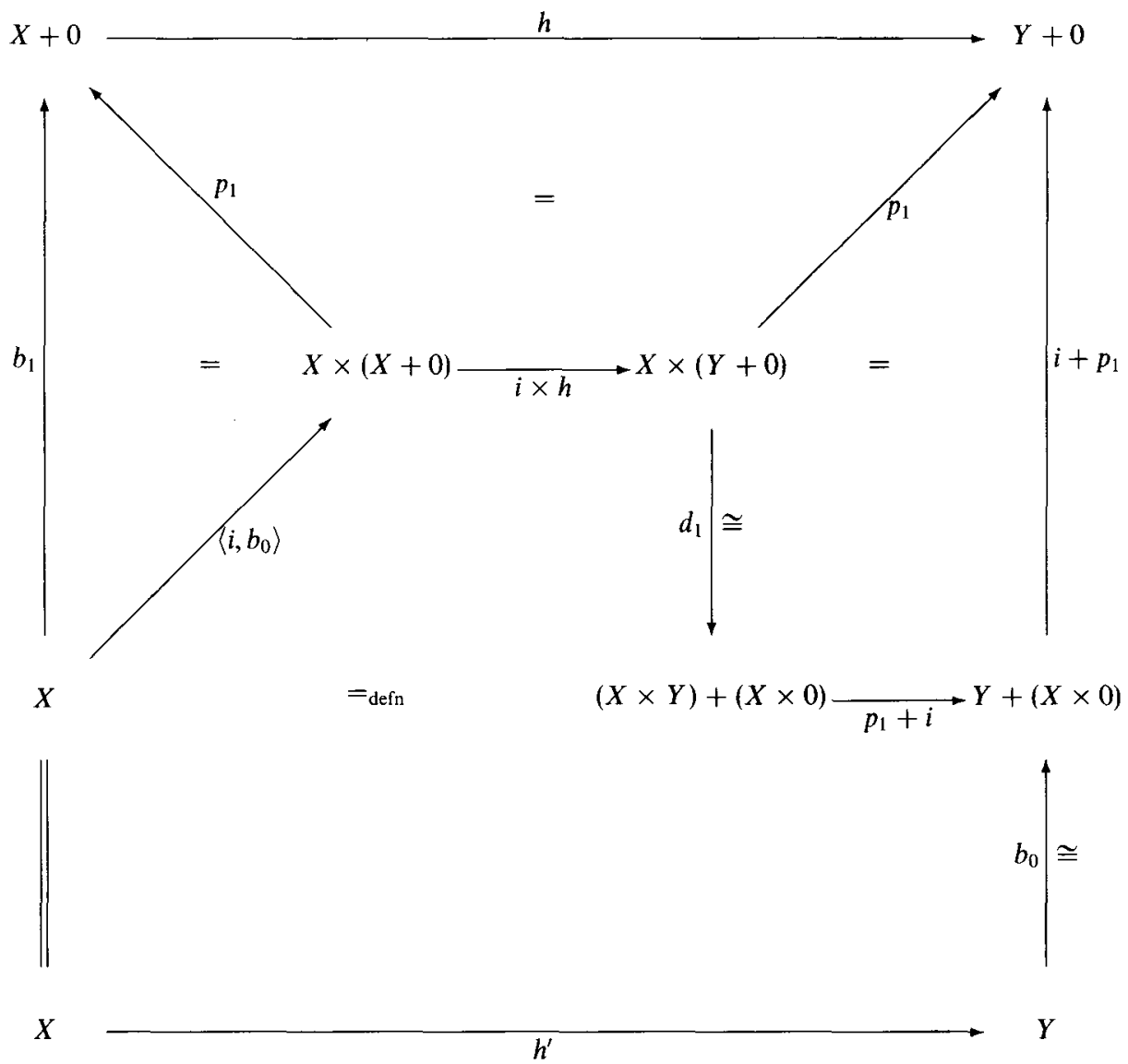

Fig. 6 .

A solid object is one that has a (necessarily unique) map from the final preinitial 0 , or, equivalently, has $X+0 \equiv X$. If there is a final preinitial, there is an obvious distributive functor $S$ that reflects $\mathbf{X}$ faithfully into the full subcategory $\operatorname{Solid}(\mathbf{X})$ of solid objects. The category Solid $(\mathbf{X})$ is isomorphic to the coslice category $0 / \mathbf{X}$, which is clearly solid. Similarly, $\operatorname{Pr}(\mathbf{X})$ is isomorphic to the slice category $\mathbf{X} / 0$ and so has a coreflection $P$.

Proposition 3.6. If $\mathbf{X}$ is a distributive category with a final preinitial 0 , then

(i) $P=-\times 0: \mathbf{X} \longrightarrow \operatorname{Pr}(\mathbf{X})$ is a distributive coreflection,

(ii) $S=-+0: \mathbf{X} \longrightarrow \operatorname{Solid}(\mathbf{X})$ is a faithful distributive reflection to a solid full subcategory,

(iii) $\langle P, S\rangle: \mathbf{X} \longrightarrow \operatorname{Pr}(\mathbf{X}) \times \operatorname{Solid}(\mathbf{X})$ is a fully faithful distributive subdirect decomposition.

The fact that the slicing and coslicing give rise to distributive functors relies heavily on the fact that $\mathbf{X}$ is distributive. To prove that the decomposition is full, given $h$ : $X+0 \longrightarrow Y+0$ and that $P(X) \leq P(Y)$, the diagram in Figure 6 is useful. 


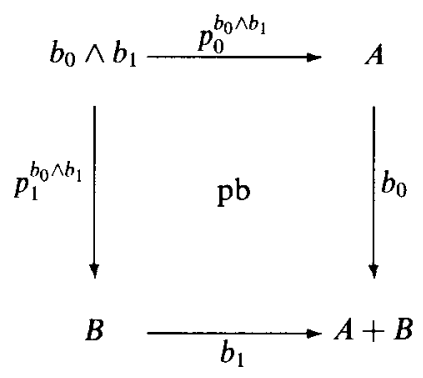

Fig. 7.

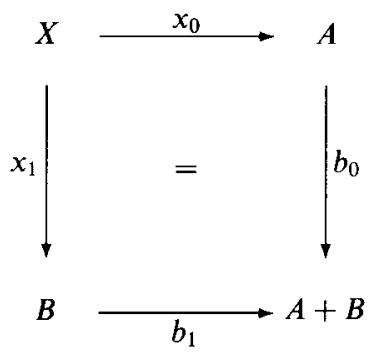

Fig. 8 .

Its purpose is to give the definition of a map $h^{\prime}: X \longrightarrow Y$ such that $h=h^{\prime}+i$. To this end, note that $Y \equiv Y+X \times 0$, as $P(X) \leq P(Y)$. This means that the lowest polygon can be used to define $h^{\prime}$.

The functor -+0 is the solidification functor. We shall return shortly to the problem of performing a solidification when no final preinitial is present.

Distributive categories can be viewed as full subcategories of the product of a solid distributive category and a prelattice. In fact, we will show that a distributive category is always a full subcategory of the product of a topos and a prelattice.

\subsection{Disjointness}

Coproducts are disjoint (respectively quasi-disjoint) when all pullbacks of the form shown in Figure 7 exist and $b_{0} \wedge b_{1}$ is initial (respectively preinitial).

Observe that if the square in Figure 8 commutes, then certainly $X$ is preinitial. Thus, if there is a largest preinitial with a map to both $A$ and $B$, that will be the pullback. When the category is quasi-solid, this largest preinitial exists and is $P(A) \times P(B)$. This means that coproducts are quasi-disjoint. The converse is also true, as the final preinitial is necessarily the pullback of the coproduct embeddings of $1+1$.

Proposition 3.7. A distributive category is (quasi-) solid if and only it has (quasi-) disjoint coproducts. 


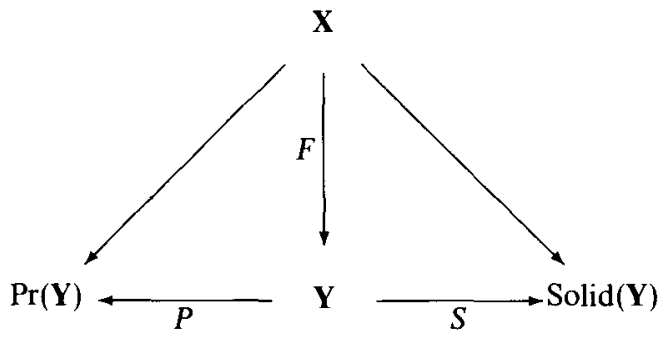

Fig. 9.

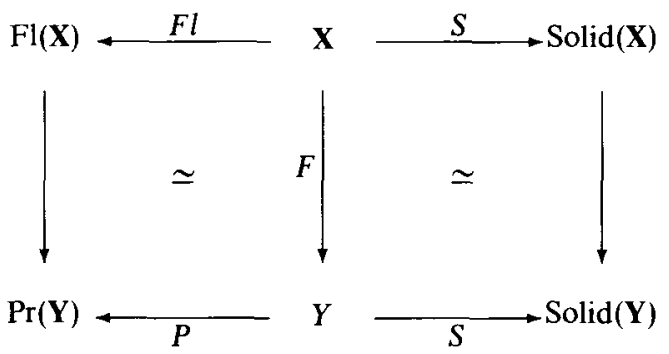

Fig. 10 .

\subsection{Solidification}

To adjoin an initial object to a distributive category is trivial: one simply adds a new object $\emptyset$ together with a unique map to each object (the composition is then forced). However, adding a final preinitial is considerably more delicate. I shall now describe how this can be done using the general version of solidification.

Suppose that $\mathbf{X}$ is an arbitrary distributive category and $F: \mathbf{X} \longrightarrow \mathbf{Y}$ is a distributive functor to $\mathbf{Y}$, a quasi-solid distributive category. As $\mathbf{Y}$ can be subdirectly decomposed, we have the diagram in Figure 9. If $F l(X)$ is the reflection of $\mathbf{X}$ to prelattices and Solid(X) is the reflection to solid distributive categories, we can factorize $F \circ P$ and $F \circ S$, respectively, through these categories (Figure 10).

The quasi-solid completion of $\mathbf{X}$ can be constructed as the smallest full distributive subcategory of $\mathrm{Fl}(\mathbf{X}) \times \operatorname{Solid}(\mathbf{X})$ containing all objects of the form $\left\langle P_{\mathbf{X}}(X), S_{\mathbf{X}}(X)\right\rangle$, for $X \in \mathbf{X}$, and the final preinitial $\left\langle P_{\mathbf{X}}(1), 0\right\rangle$.

Notice that this shows that the preinitial lattice of the quasi-solid completion of $\mathbf{X}$ is equivalent to the flattening of $\mathbf{X}$ by reflection to preorders. This indicates the information the preinitial prelattice of a distributive category may contain.

It remains to construct Solid(X). This construction has two stages. First, an initial object $\emptyset$ is adjoined (as discussed above). Second, all maps of the form $b_{0}: X \longrightarrow X+P$ (where $P$ is preinitial), called thin detachments, are formally inverted to obtain a calculus of left fractions (Gabriel and Zisman 1967).

Now, let $\Sigma$ denote the class of thin detachments of a distributive category $\mathbf{X}$, which has an initial object. It is easy to check that the following proposition holds. 
Proposition 3.8. In any distributive category, $\Sigma$ admits a calculus of left fractions.

It follows that $S_{\mathbf{X}}: \mathbf{X} \longrightarrow \mathbf{X}\left(\Sigma^{-1}\right)$ preserves finite colimits and $\mathbf{X}\left(\Sigma^{-1}\right)$ has coproducts. It is also easily seen that $S_{\mathbf{X}}$ preserves and $\mathbf{X}\left(\Sigma^{-1}\right)$ has finite products and is distributive. Also, as each $b_{0}: \emptyset \longrightarrow \emptyset+P$ is inverted, it is clear that $\mathbf{X}\left(\Sigma^{-1}\right)$ has a strict final preinitial object, and so is solid.

In general, the category of fractions construction can turn a locally small category into one that is not locally small. This does not happen in this case, because of the following lemma.

Lemma 3.9. If $f: X \longrightarrow X+P$ and $g: X \longrightarrow X+P^{\prime}$, and $P$ and $P^{\prime}$ are preinitial, then there is a $g^{\prime}: X \longrightarrow X+P$ such that, in $\mathbf{X}\left(\Sigma^{-1}\right), g \cdot b_{0}^{-1}=g^{\prime} \cdot b_{0}^{-1}$.

Proof. Define $g^{\prime}$ by $g^{\prime}=\langle f, g\rangle \cdot \gamma \cdot\left\langle p_{1}+p_{0}\right\rangle$, where $\gamma:(X+P) \times\left(X+P^{\prime}\right) \longrightarrow(X \times X)+$ $\left(P \times P^{\prime}\right)$ is the evident isomorphism. It is easy to check that $g^{\prime}$ has the desired property.

$S_{\mathbf{X}}: \mathbf{X} \longrightarrow \mathbf{X}\left(\Sigma^{-1}\right)$ is faithful, as all inverted maps are bijections. Furthermore, $\mathbf{X}\left(\Sigma^{-1}\right)$ is the "solidification" of $\mathbf{X}$, as clearly any such process must at least invert the maps of $\Sigma$. This gives the folowing theorem.

Theorem 3.10. $S_{\mathbf{X}}: \mathbf{X} \longrightarrow \mathbf{X}\left(\Sigma^{-1}\right) \equiv \operatorname{Solid}(\mathbf{X})$ is a faithful distributive functor to a solid distributive category, and is such that given any distributive functor

$$
F: \mathbf{X} \longrightarrow \mathbf{Y}
$$

where $\mathbf{Y}$ is a solid distributive category, there is a unique functor $F^{*}: \operatorname{Solid}(\mathbf{X}) \longrightarrow \mathbf{Y}$ with $S_{\mathbf{X}} \circ F^{*}=F$.

If $\mathbf{X}$ already has a final preinitial object 0 , then $\operatorname{Solid}(\mathbf{X})$ is equivalent to the full subcategory of solid objects, as $b_{0}: X \longrightarrow X+0$ is in $\Sigma$, and any $b_{0}: X+0 \longrightarrow(X+0)+P$ is already an isomorphism.

Corollary 3.11. If $\mathbf{X}$ has a final preinitial, $\operatorname{Solid}(\mathbf{X}) \equiv \mathbf{X}\left(\Sigma^{-1}\right) \equiv 0 / \mathbf{X}$.

If $F: \mathbf{X} \longrightarrow \mathbf{Y}$ is a faithful distributive functor and $f \cdot b_{0}^{-1}, g . b_{0}^{-1}: A \longrightarrow B$ in $\operatorname{Solid}(\mathbf{X})$, then, using the lemma above, we may alter $g$ to a $g^{\prime}$ having the same codomain as $f$ in X. If $f \cdot b_{0}^{-1} \neq g . b_{0}^{-1}$, then $f \cdot b_{0}^{-1} \neq g^{\prime} \cdot b_{0}^{-1}$. But now $S_{\mathbf{Y}}(F(f)) \neq S_{\mathbf{Y}}\left(F\left(g^{\prime}\right)\right)$, so that certainly $\operatorname{Solid}(F)\left(f \cdot b_{0}^{-1}\right) \neq \operatorname{Solid}(F)\left(g . b_{0}^{-1}\right)$, showing that $\operatorname{Solid}(F)$ is faithful whenever $F$ is.

Corollary 3.12. If $F: \mathbf{X} \longrightarrow \mathbf{Y}$ is a faithful distributive functor between distributive categories, then $\operatorname{Solid}(F): \operatorname{Solid}(\mathbf{X}) \longrightarrow \operatorname{Solid}(\mathbf{Y})$ is faithful.

In case one is tempted to believe that solid distributive categories have a simple structure, consider the following example, which provides a solid distributive category that cannot be embedded in a topos.

Example: $\mathbf{D}[g]$ cannot be embeded in a topos.

Consider the free distributive category $\mathbf{D}[g]$ generated by the condition that $g: X \longrightarrow$ $Y+1$ equalizes $i+b_{0}, i+b_{1}: Y+1 \longrightarrow Y+(1+1)$ (that is, $\left.g \cdot\left\langle i+b_{0}\right\rangle=g .\left\langle i+b_{1}\right\rangle\right)$. This category is non-trivial and has no preinitial objects. This can be seen, as there is a model in $\operatorname{Set}_{f}^{*} \times \mathbf{2}$ (where Set $_{f}^{*}$ is the category of finite sets without the empty set and $\mathbf{2}$ is $!: 0 \longrightarrow 1)$ given by $g=\left(b_{0}, !\right):(1,1) \longrightarrow(1,0)+(1,1)$. Notice that there is no map from $(1,1)$ to $(1,0)$, and the distributive category $\operatorname{Set}_{f}^{*} \times 2$ has no preinitials.

As $\mathbf{D}[g]$ has no preinitials, adjoining an initial object $\emptyset$ makes this category solid. 


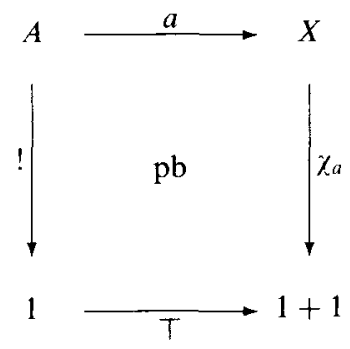

Fig. 11 .

However, in any topos it is easy to see that such a $g$ must factor through the embedding $b_{0}$. Therefore, $\mathbf{D}[\mathrm{g}]$ does not have a full and faithful embedding into a topos.

\section{Embedding theorems}

In general, when one has an elementary condition, such as the distributive law, it is traditional to consider the effect of requiring it to hold in the slice categories as well as the category itself. One then says that the category locally satisfies the condition. A variant of this is to ask that the condition be satisfied in some specific slices.

The object $1+1$ in distributive categories forms a boolean algebra and is a natural candidate from which to build an internal boolean logic. The subobjects that can be described in this logic should be thought of as the "recursive" subobjects of the setting. More precisely, they are subobjects for which there is a recognizer, where a subobject $a: A \longrightarrow X$ has a recognizer $\chi_{a}: X \longrightarrow 1+1$ whenever the square in Figure 11 is a pullback. Here $T=b_{0}, \perp=b_{1}: 1 \rightarrow 1+1$. The recognizer of a subobject is distinct from the characteristic map. The latter is a map to a subobject classifier, which always exist: recognizers, of course, will not always exist. As $1+1$ is a subobject of any (more general) subobject classifier, this internal logic will always be a restriction of any more general logic.

This "recognition" logic will only give information about the subobject structure of the category if subobjects corresponding to recognizers actually exist. We may guarantee this by requiring that the distributive category is local at $1+1$, that is $\mathbf{X} / 1+1$ is distributive. Such a category has the necessary subobject structure to carry this logic and is called a recognizable distributive category.

\subsection{Local properties}

A map $f: X \rightarrow Y$ is quarrable if one can pullback along it. If, in addition, this pulling back preserves coproducts, that is $f^{*}: \mathbf{X} / Y \longrightarrow \mathrm{X} / X$ preserves coproducts, then $f$ is said to be coproductive. Pullbacks and compositions of coproductive maps are also coproductive.

An object $X$ is said to be distributive if slicing at that object gives a distributive category, $\mathbf{X} / X$. An object is distributive if and only if every map into that object is coproductive. Subobjects of distributive objects are distributive. 


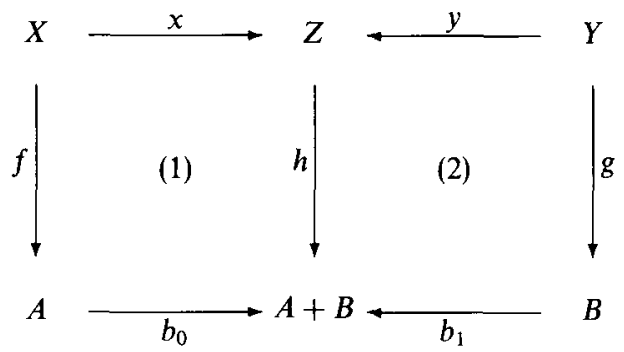

Fig. 12.

In any distributive category, the final object 1 is always distributive. As mentioned above, to secure the correspondence between recognizers and subobjects, it suffices to require that $1+1$ be distributive. We shall interpret this as a requirement on that coproduct.

A coproduct $\left(A+B, b_{0}, b_{1}\right)$ is quasi-extensive when the embeddings are quarrable, and given any diagram of the form shown in Figure 12, where (1) and (2) are pullbacks, the top row $(Z, x, y)$ is a coproduct. It is said to be extensive if, in addition, whenever $(Z, x, y)$ is a coproduct, (1) and (2) must be pullbacks.

If every coproduct is extensive, the category is said to be extensive: this situation will be discussed in the next sections.

Notice that if $\left(A+B, b_{0}, b_{1}\right)$ is a quasi-extensive coproduct, then certainly the top row of the diagram will also be a quasi-extensive coproduct. I shall refer to $(Z, x, y)$ as the coproduct induced by $h$ over $\left(A+B, b_{0}, b_{1}\right)$.

Lemma 4.1. $A+B$ is distributive if and only if $\left(A+B, b_{0}, b_{1}\right)$ is quasi-extensive, $b_{0}$ and $b_{1}$ are coproductive, and $A$ and $B$ are distributive.

If $A+B$ is distributive, then certainly $A$ and $B$ will be, as they are subobjects. The maps $b_{0}$ and $b_{1}$ are coproductive, as any map to a distributive object is. Finally, pulling back $b_{0}+b_{1}$ along any map to $A+B$ shows that $\left(A+B, b_{0}, b_{1}\right)$ is quasi-extensive.

To obtain the reverse implication, it is necessary to show that any map into $A+B$ is coproductive. To show this, we certainly need to show that such a map is quarrable. The pullback along such a map is formed by using the coproduct components induced over $\left(A+B, b_{0}, b_{1}\right)$. This gives two pullbacks over $A$ and $B$ : as these objects are distributive, these pullbacks may be formed. The sum of these pullbacks is then a pullback for the whole. This construction of the pullback shows how the coproductivity of the component maps can be reconstituted into the coproductivity of the whole.

We note that, in general, pulling back $f+g$ along $b_{0}$ does not quite recapture the first component. This is the essential subtlety that we are being careful to capture.

Lemma 4.2. If the embedding $b_{0}: A \longrightarrow A+B$ is locally coproductive and $f+g$ : $X+Y \longrightarrow A+B$, then $b_{0} \wedge\langle f+g\rangle \equiv X+P$, where $P$ is a preinitial.

\subsection{Recognition properties}

A recognizable distributive category has a final preinitial given by the pullback $T \wedge \perp$. Thus, the category has quasi-disjoint coproducts. 


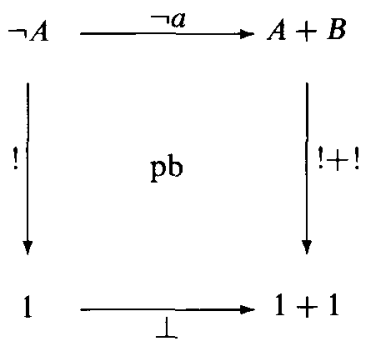

Fig. 13.

A subobject $a: A \longrightarrow X$ is detachable if there is another subobject $b: B \longrightarrow X$ with $\langle a \mid b\rangle: A+B \longrightarrow X$ an isomorphism. It is complemented if it is detachable and (preinitial) saturated, that is any preinitial $P$ with $P \longrightarrow X$ has $P \longrightarrow A$. As there is a final preinitial, $a: A \longrightarrow X$ is saturated if and only if $P(A) \simeq P(X)$. A subobject of $X$ can always be saturated by adding $P(X)$.

Lemma 4.3. In a recognizable distributive category, if $a: A \longrightarrow X$ is detachable, there is a unique (to isomorphism) complemented subobject, $\neg a: \neg A \longrightarrow X$, such that $\langle a \mid \neg a\rangle$ : $A+\neg A \longrightarrow X$ is an isomorphism.

If $\langle a \mid b\rangle: A+B \longrightarrow X$ is an isomorphism, we may form the square in Figure 13 by pullback. $(\neg A, \neg a)$ is both saturated and detachable (the latter because $A+\neg A \equiv$ $A+(B+P) \equiv X)$. Furthermore, $\neg a: \neg A \longrightarrow X$ is independent of the choice of $b: B \longrightarrow X$, so any $b: B \longrightarrow X$ with $A+B \equiv X$ factors uniquely through $\neg a: \neg A \longrightarrow X$. In fact, as $\neg A \equiv B+P$ for any such $B$, we have $\neg A \equiv B$ when $B$ is saturated.

This shows that the lattice of complemented subobjects of $X, \operatorname{sub}_{\mathrm{c}}(X)$, is a boolean algebra. In fact, we have all but shown the following proposition.

Proposition 4.4. In a recognizable distributive category $X, 1+1$ is a boolean algebra, and $T^{*}: \mathbf{X}(X, 1+1) \longrightarrow \operatorname{Sub}_{\mathrm{c}}(X)$ is an isomorphism of boolean algebras.

This means complemented subobjects are represented by unique recognizers, recognizers are represented by unique (to isomorphism) complemented subobjects, and these representations are mutually inverse. The logic based on recognizers makes subobject sense when this happy coincidence reigns.

\subsection{Characterizations of recognition}

A coproduct $\left(A+B, b_{0}, b_{1}\right)$ is saturated when each of its components is. Every such coproduct occurs as a splitting of $!+$ ! over $(1+1, T, \perp)$. This immediately gives the following lemma.

Lemma 4.5. In a recognizable distributive category all saturated coproducts have locally coproductive embeddings and are quasi-extensive.

Notice, now, that all components of a coproduct that contain an element (map from 1) are necessarily saturated. Thus, in a recognizable distributive category, any coproduct of final objects is locally distributive by the following structural induction: 1 is locally 


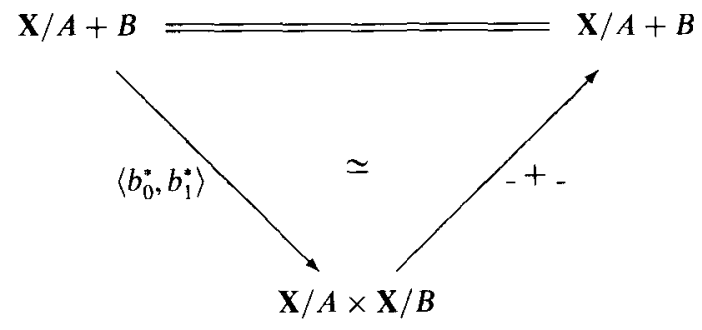

Fig. 14.

distributive and $(1+\ldots+1)+(1+. .+1)$ is locally distributive if and only if its components are locally distributive (inductive assumption), its embeddings are locally coproductive, and the coproduct is quasi-extensive. The latter two properties are given by the previous lemma and the remark which started this paragraph. This leads to the following proposition.

Proposition 4.6. If $\mathbf{X}$ is distributive, the following are equivalent

(i) $\mathbf{X}$ is recognizable,

(ii) $(1+1, T, \perp)$ is quasi-extensive, with each embedding locally coproductive,

(iii) every saturated coproduct is splitting, with each embedding locally coproductive,

(iv) $\mathbf{X} / 1+\ldots+1$ distributive for all non-empty coproducts of the terminal object.

A coproduct is quasi-extensive if and only if the diagram in Figure 14 commutes to equivalence. When this is the case, $\left\langle b_{0}^{*}, b_{1}^{*}\right\rangle$ includes $\mathbf{X} / A+B$ into $\mathbf{X} / A \times \mathbf{X} / B$ as the full reflexive subcategory with objects saturated pairs $(X, Y)$, that is, having $P(X) \equiv P(Y)$. This gives the following proposition.

Proposition 4.7. If $X$ is distributive, the following are equivalent:

(i) $\mathbf{X}$ is recognizable distributive,

(ii) $\left\langle T^{*}, \perp^{*}\right\rangle: \mathbf{X} / 1+1 \longrightarrow \mathbf{X} \times \mathbf{X}$ is a coproductive inclusion of a reflexive subcategory,

(iii) for every saturated coproduct, $\left\langle b_{0}^{*}, b_{1}^{*}\right\rangle: \mathbf{X} / A+B \longrightarrow \mathbf{X} / \boldsymbol{A} \times \mathbf{X} / B$ is a coproductive inclusion of a reflexive subcategory.

\subsection{Extensive categories}

A recognizable distributive category still does not necessarily have every detachable map complemented, as not every detachable map need be saturated. This defect can be remedied by passing to the solid full subcategory. A solid recognizable distributive category is an extensive distributive category. However, this is not the preferred definition. ${ }^{\dagger}$

$\uparrow$ Gordon Monro (Sydney Category Seminars 1988), following the work of Schanuel, explicitly studied the properties of distributive categories satisfying the extensive property without the assumption of finite completeness. He realized that they had very attractive properties, especially from the point of view of studying recursive properties, exactly because detachable and complemented coincide. In 1990, I talked at the Sydney Category Seminar about the recognizable completion and Gordon, realizing the connection, made his notes available. The current treatment is greatly influenced by those notes. 


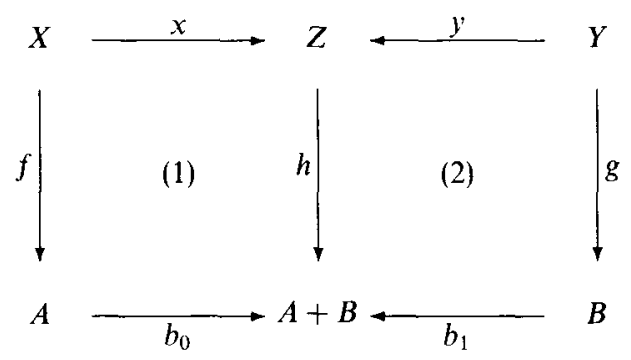

Fig. 15.

An extensive distributive category is a cartesian category with binary coproducts such that for any $A$ and $B$

$$
\text { - }_{-}: \mathrm{X} / A \times \mathrm{X} / B \stackrel{\cong}{\longrightarrow} \mathrm{X} / A+B \text {. }
$$

This can be simplified to the equivalent requirement that

$$
+_{-}: \mathbf{X} \times \mathbf{X} \stackrel{\cong}{=} \mathbf{X} / 1+1
$$

These conditions can be reexpressed as: $\left(A+B, b_{0}, b_{1}\right)$ is an extensive coproduct for any $A$ and $B$; and $(1+1, \top, \perp)$ is an extensive coproduct, respectively. For proving things about extensive distributive categories, the former condition is more useful, while, for proving that something is an extensive distributive category, the latter condition is the more useful.

Diagrammatically, the former condition is shown in Figure 15, where (1) and (2) are pullbacks if and only if the top row, $(Z, x, y)$, is a coproduct. This condition was, for example, used explicitly in the development of Mackey functors by Dress (1973) and Lintner (1976). However, it was also assumed that the categories were finitely complete, making them Schanuel/Lawvere distributive.

If $\mathbf{X}$ is a solid distributive category, then, whenever $A+B$ is locally distributive, $\left(A+B, b_{0}, b_{1}\right)$ will be extensive. If $1+1$ is locally distributive, then, as every coproduct is obtained as the coproduct induced by $!+!: A+B \longrightarrow 1+1$ over $(1+1, \top, \perp)$, every coproduct will be extensive. Thus, a solid recognizable distributive category is an extensive distributive category.

Proposition 4.8. When $X$ has finite products and binary coproducts, the following are equivalent:

(i) $\mathbf{X}$ is extensive,

(ii) $\mathbf{X}$ is solid recognizable distributive,

(iii) $\mathbf{X}$ is solid and has every coproduct quasi-extensive,

(iv) every coproduct is extensive,

(v) $(1+1, T, \perp)$ is extensive.

One should not forget that the squares shown in Figure 16 are always pullbacks. The left square can be used to show that when coproducts are quasi-extensive, the category is automatically distributive. Also observe that if every coproduct is extensive, pullbacks will preserve and be created by coproducts. More precisely, we have the following lemma.

Lemma 4.9. In an extensive distributive category, the pullbacks in Figure 17 exist if and 


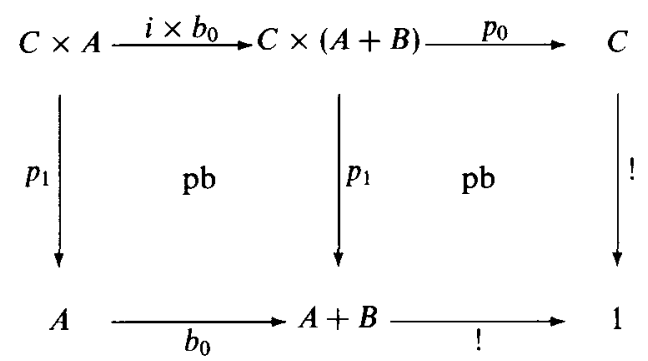

Fig. 16.
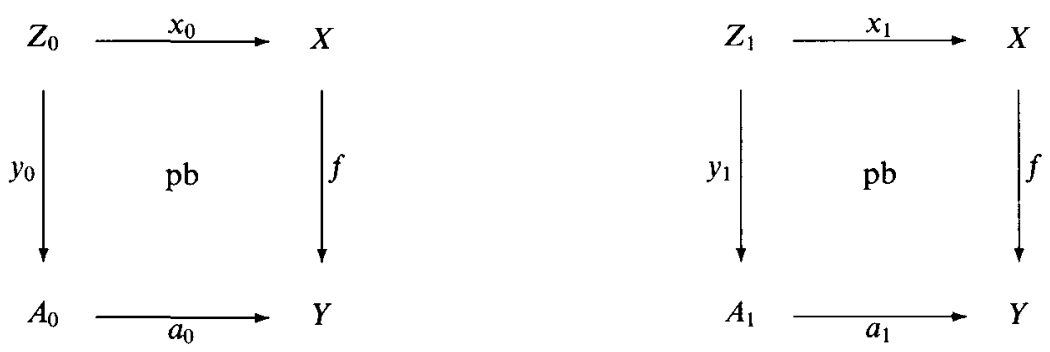

Fig. 17.

only if the pullback in Figure 18 exists, where Figure 19 holds, with $x_{0}^{\prime} \cdot x=x_{0}$ and $x_{1}^{\prime} \cdot x=x_{1}$.

The proof of this is straightforward and simply uses the fact that any map to $A_{0}+A_{1}$ results in the domain being split into a coproduct.

A coproduct is extensive if and only if the two functors $\left\langle b_{0}^{*}, b_{1}^{*}\right\rangle$ and $+_{-}$are adjoint equivalences. In this case $\left\langle b_{0}^{*}, b_{1}^{*}\right\rangle$ is forced to be coproductive, and this allows the results to be stated in terms of this functor.

Proposition 4.10. When $X$ has finite products and binary coproducts, the following are equivalent:

(i) $\mathbf{X}$ is a solid recognizable distributive category,

(ii) $\mathrm{X} / A \times \mathrm{X} / B \simeq \mathrm{X} / A+B$ for every $A$ and $B$ by ${ }_{-}+$

(iii) $\mathbf{X} \times \mathbf{X} \simeq \mathbf{X} / 1+1$ by $_{-}+$.

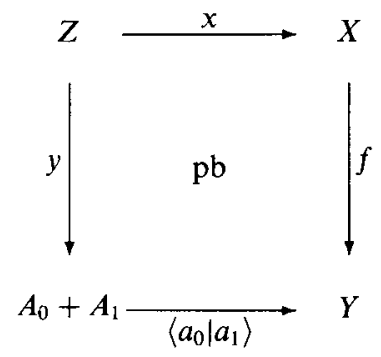

Fig. 18. 


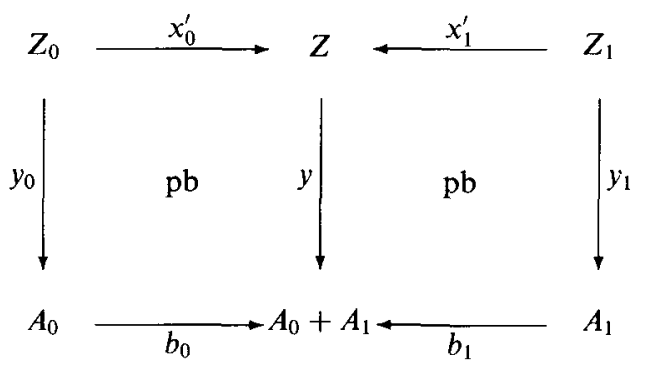

Fig. 19.

These are the forms of the extensive property introduced by Steve Schanuel. ${ }^{\dagger}$

In an extensive distributive category, a coproduct can be characterized as a regular epic family of mutually disjoint monic maps. Furthermore, monic maps from coproducts are given by mutually disjoint monic families.

Proposition 4.11. The map $\left\langle a_{0} \mid a_{1}\right\rangle: A_{0}+A_{1} \longrightarrow X$ is monic if and only if $a_{0}$ and $a_{1}$ are monic and disjoint.

Notice that we are not guaranteed that the pullback $a_{0} \wedge a_{1}$ exists in an extensive distributive category. The fact that it does is a component of the lemma. This makes the result a little more delicate than it may seem at first sight.

If $\left\langle a_{0} \mid a_{1}\right\rangle$ is monic, then certainly $a_{0}$ and $a_{1}$ must be monic and disjoint. For the converse, we should consider the pullback of $\left\langle a_{0} \mid a_{1}\right\rangle$ over itself. This exists only as the pullbacks over each component do and

$$
\left(a_{0} \wedge a_{0}\right)+\left(a_{0} \wedge a_{1}\right)+\left(a_{1} \wedge a_{0}\right)+\left(a_{1} \wedge a_{1}\right)=A_{0}+0+0+A_{1}=A_{0}+A_{1},
$$

from which the result is immediate.

As equalizers are pullbacks over diagonals, when an extensive distributive category has all diagonal maps detachable, it is finitely complete, and, therefore, is a Schanuel/Lawvere distributive category.

Proposition 4.12. An extensive distributive category with all objects decidable is finitely complete.

A functor of extensive categories should preserve the ingredients of the extensive property. In particular, a functor must preserve pullbacks along detachments. The following lemma contains a nice observation.

Lemma 4.13. A distributive functor between extensive distributive categories preserves pullbacks over detachable subobjects.

Thus, we take the appropriate 2-category to be extensive distributive categories with distributive functors. It should be noted that we do not therefore guarantee the preservation of arbitrary finite limits: a fact to which we will return.

$\dagger$ In fact, both Schanuel and Monro demanded that there be an initial object and $\mathbf{X} / 0 \simeq \mathbf{1}$ (which is the requirement that 0 is a strict initial object). Both requirements are redundant: the initial object is $T \wedge \perp$, and it is necessarily strict. 


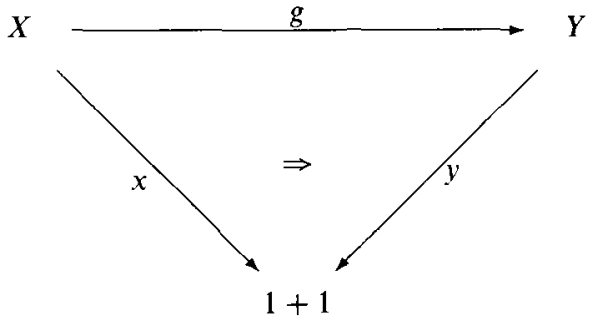

Fig. 20.

\subsection{Recognizable completion}

The purpose of this section is to describe the construction of the free recognizable distributive category from a distributive category. The construction has a number of important ramifications. Its purpose in this discussion is to provide the crucial link between distributive and extensive categories.

Let $\mathbf{X}$ be a distributive category, then denote by $\operatorname{Rc}(\mathbf{X})$ the following category:

Objects: The objects are maps $x: X \longrightarrow 1+1$ of $\mathbf{X}$,

Maps: The maps are "almost commuting" triangles (see Figure 20), where $\langle x, g \cdot y\rangle . \Rightarrow=T$, where $\Rightarrow$ is Boolean implication.

Define $r c_{x}(g):=\langle x, g\rangle \cdot d_{0} \cdot\left\langle p_{1}+!\right\rangle$. Then two maps $g_{1}, g_{2}: f \longrightarrow h$ are equivalent if and only if $r c_{x}\left(g_{1}\right)=r c_{x}\left(g_{2}\right)$.

Notice that the equivalence is to ensure that two maps are identified when their behaviors over the characterized parts are the same.

Theorem 4.14. For any distributive category $\mathbf{X}$, the recognizable completion $R c(X)$ is a recognizable distributive category that includes $\mathbf{X}$ distributively, fully, and faithfully by

$$
R_{\mathbf{X}}: \mathbf{X} \longrightarrow \operatorname{Rc}(\mathbf{X}) ; X \longrightarrow !_{X} \cdot \top .
$$

Furthermore, given any distributive functor $F$ from $\mathbf{X}$ to a recognizable distributive category $\mathbf{Y}$, there is a unique to unique equivalence recognizable distributive functor

$$
\widetilde{F}: \operatorname{Rc}(\mathbf{X}) \longrightarrow \mathbf{X}
$$

such that $R \circ \widetilde{F} \simeq F$.

The construction is actually a limited equalizer completion, and there are various reasons for considering this restriction. The main reasons derive from the economical nature of the construction.

If $\mathbf{X}$ is a category whose map equality predicate is decidable (externally), then $\operatorname{Rc}(\mathbf{X})$ also has a decidable map equality predicate. This can be seen directly from the construction: two maps $g_{1}, g_{2}: x \longrightarrow y$ are equal precisely if $r c_{x}\left(g_{1}\right)=r c_{x}\left(g_{2}\right)$ in $\mathbf{X}$. This gives the following corollary.

Corollary 4.15. $\mathbf{X}$ has a decidable map equality predicate if and only if $\operatorname{Rc}(\mathbf{X})$ has its map equality predicate decidable. 


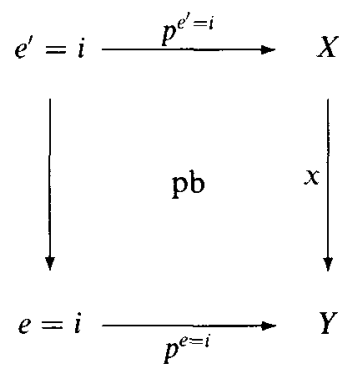

Fig. 21.

The "only if" arises because $\mathbf{X}$ can be fully and faithfully embedded in $\operatorname{Rc}(\mathbf{X})$. This direction is particularly useful in proving the undecidability of $\mathbf{X}$, as one can often obtain such a proof more easily using the recognizable completion. The positive direction is also useful as the constructive decidability of $\mathbf{X}$ will often be easier to obtain than for the completion.

The proof of the main theorem may be accomplished in two stages. First by forming the category $\mathbf{X} / \mathscr{B}$, where $\mathscr{B}$ is the boolean algebra on $1+1$ regarded as an internal cartesian category. This is certainly a distributive category. Next one quotients this category by the relation $g_{1} \sim_{\mathrm{rc}} g_{2}: x \longrightarrow y$ if and only if $r c_{x}\left(g_{1}\right)=r c_{x}\left(g_{2}\right)$. Checking that this results in a category of the desired form is lengthy but straightforward. To obtain the desired universal property, one uses $\mathbf{X} / / \mathscr{B}$ to obtain a functor, and notes that this functor factors uniquely through $(\mathbf{X} / / \mathscr{B}) / \sim_{\text {rc }}$.

In fact, a good deal more can be taken through this construction: for example, if $\mathbf{X}$ has list-arithmetic, then $\operatorname{Rc}(\mathbf{X})$ will have list arithmetic. This will be reported in detail in a later paper, and is beyond the scope of this introduction.

A useful observation, which is needed in the construction of $\mathbf{X} / / \mathscr{B}$, is that the functor from the initial distributive category, that is finite sets without the empty set Set $_{f}^{*}$, preserves all the finite colimits and limits that exist. The latter fact is the more surprising and useful, as this assures us that $\mathscr{B}$ is indeed an internal cartesian category. Furthermore, it is a consequence of a quite general resuit.

Proposition 4.16. If $\mathbf{Y}$ is a distributive category with all regular monics coretractions and all objects decidable, then any product preserving functor from $\mathbf{Y}$ preserves all limits.

As has been mentioned already, an object is decidable in a distributive category if its diagonal map is detachable. A distributive category with every regular monic either an isomorphism or detachable will have every regular monic a coretraction when every object is inhabited (has an element). Clearly Set ${ }_{j}^{*}$ is such a category. Also, distributive categories formed as full subcategories generated by inhabited subsets of a natural number object have this property.

To prove this result, we shall use the following technical lemma.

Lemma 4.17. If $e^{\prime} . x=x . e$, where $x$ is monic and $e^{\prime}$ and $e$ are idempotent, then the square in Figure 21 is a pullback.

Proof. Let $(X,(f, g))$ be such that $f . x=g . p^{e=i}$, then $f . x . e=f . x$, so $f . e^{\prime} \cdot x=f . x$, giving 


$$
\begin{aligned}
& f=g \longrightarrow p^{f=g} \longrightarrow X \\
& \left\langle p^{f=g} . f, p^{f=g}\right\rangle \mid \quad \mathrm{pb} \quad\langle\langle f, g, i\rangle \\
& Y \times X \underset{\left\langle p_{0}, p_{0}, p_{1}\right\rangle}{\longrightarrow} Y \times Y \times X
\end{aligned}
$$

Fig. 22.

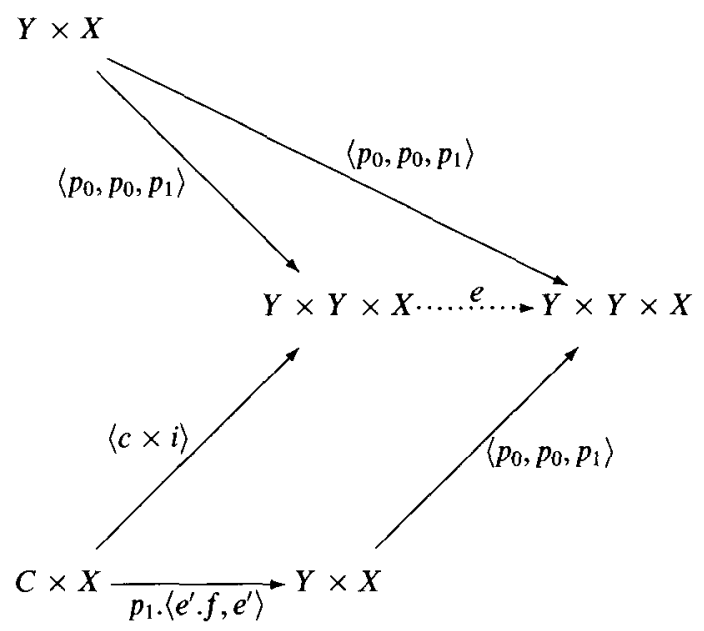

Fig. 23.

$f . e^{\prime}=f$, as $x$ is monic. Thus, $f$ factors through $p^{e^{\prime}=i}$ uniquely, showing that this is a pullback.

The point of the lemma is that equalizers of idempotents with identities are always preserved by any functor (they are absolute limits). So, provided the fact that $x$ is monic is preserved by a functor, this pullback will necessarily be preserved.

Corollary 4.18. If, in the above, $x$ is a section, the above square is an absolute pullback.

This can now be used to obtain the proof of the proposition.

Proof. (of 4.16) As finite products are preserved, it suffices to show that equalizers are also preserved. The equalizer of $f$ and $g$ can be expressed by requiring the square in Figure 22 to be a pullback.

Notice that both $\langle f, g, i\rangle$ and $\left\langle p_{0}, p_{0}, p_{1}\right\rangle$ are sections. $p^{f=g}$ is certainly regular monic, as it is the equalizer of $f$ and $g$, so, by assumption, we have a retraction of $p^{f=g}$, and an associated idempotent on $e^{\prime}: X \longrightarrow X$. Define $e: Y \times Y \times X \longrightarrow Y \times Y \times X$ by the diagram in Figure 23, where $(Y \times Y,\langle i, i\rangle, c)$ is the coproduct giving the detachment of the diagonal. Clearly $e$ splits through $\left\langle p_{0}, p_{0}, p_{1}\right\rangle$. 
I claim $\langle f, g, i\rangle . e=e^{\prime} \cdot\langle f, g, i\rangle$, so the lemma above can be applied. To see this, follow through the maps on $f=g$ and $\neg f=g$.

Thus, we have that $\mathscr{B}$ is an internal cartesian category, whence we can prove that every distributive category has a faithful embedding in an extensive distributive category. The argument is as follows: the completion procedure allows us to pass fully and faithfully to a recognizable distributive category, whence, by solidification, faithfully to the full subcategory of strict objects. This full subcategory is extensive distributive, and we obtain the faithful passage from distributive to extensive distributive ${ }^{\dagger}$

It is worth noting that the recognizable completion of Set $_{f}^{*}$ is Set $_{f}$. The recognizable completion of Set $_{f}$, however, adds a new final preinitial object. This means that the procedure is nowhere idempotent (except on the degenerate category).

\subsection{Topos embedding}

If $\mathbf{X}$ is an extensive distributive category, it forms a site with covers given by finite coproduct decompositions. These covers contain the identity, and are stable and composable (Barr and Wells 1985), so they form a Grothendiek topology $\ddagger$ This topology is called the decomposition topology on $\mathbf{X}$ and is denoted $\mathbf{d}$. The fact that coproducts are disjoint makes it immediate that the representable functors are sheaves; that is, $\mathbf{d}$ is subcanonical.

The embedding $\mathscr{Y}: \mathbf{X} \longrightarrow$ Set $_{\mathbf{d}}^{\mathbf{X}^{\mathrm{op}}}$ is certainly full and faithful and preserves any limits that happen to be present in $\mathbf{X}$. Coproducts are also preserved, as, being covers, they become jointly epimorphic families of subobjects that are mutually disjoint (the empty set is a cover of the initial object). Furthermore, in order for the embedding to preserve coproducts, these families must certainly provide covers in the saturated topology.

Clearly, any topology stronger than $\mathbf{d}$ will result in $Y$ being a distributive functor.

$\dagger$ This faithful passage can be used to obtain many less than obvious results on distributive categories. A personal favorite is as follows:

"No non-trivial distributive category has enough fixed points."

A map $g: X \longrightarrow Y$ has a fixed point if there is an element fix $(g): 1 \longrightarrow X$ with $f(x)(g)=f(x)(g) . g$. Consider $\neg: 1+1 \longrightarrow 1+1$ and suppose that $\mathrm{fx}(\neg): 1 \longrightarrow 1+1$ exists. Embed into an extensive distributive category and form

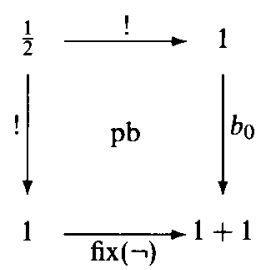

Now, $\frac{1}{2}+\frac{1}{2} \simeq 1$, yet !. $b_{0}=!$. fix $(\neg)=!$. fix $(\neg) . \neg=! . b_{0} . \neg=! . b_{1}$, which means that $\frac{1}{2}$ is preinitial. However, this means 1 is preinitial, which means that the distributive category is trivial (in the sense of every object being preinitial).

The result is of some classical importance, as it has often been assumed that "sensible computer science" settings should have enough fixed points. Yet forcing fixed points to be present implies one cannot have sensible coproducts: I am not alone in believing this is sheer stupidity!

$\ddagger$ This a pretopology in the terminology of Johnstone (1977). 
In particular, the canonical topology will have this property. This gives the following proposition.

Proposition 4.19. If $\mathrm{X}$ is extensive distributive, the Yoneda functor

$$
\mathscr{Y}: \text { Set } \longrightarrow \text { Set }_{\mathbf{j}}^{\mathbf{X}}
$$

is a distributive functor if and only if $\mathbf{j}$ is stronger than $\mathbf{d}$. Furthermore, $\mathbf{d}$ is a subcanonical topology.

This result shows that extensive distributive categories can be embedded in a topos and, furthermore, shows that the unit of the 2-adjunction to topoi is full and faithful.

Now every distributive category can be embedded fully and faithfully into a recognizable distributive category, which, in turn, can be decomposed as a subdirect product of an extensive distributive category and prelattice (with possibly no lower bound). Finally an extensive distributive category can be embedded into a topos, so we have the following corollary.

Corollary 4.20. Any distributive category has a full faithful distributive embedding into a product of a topos and a prelattice.

\section{Familial distributive categories}

An important problem for computer science applications is that of generating free distributive categories. One aspect of this problem concerns freely generating distributive categories from cartesian categories corresponding to equational theories. Surprisingly, the construction is simply the free coproduct completion, ignoring the cartesian structure: this was observed by Shu-Hao Sun and Bob Walters (Sydney Category Seminars 1990).

The generalization of this, which follows the ideas in Yves Diers thesis, is to consider categories that have familial products and equalizers. The coproduct completions of these more general categories are also distributive categories. They are characterized by each object having a finite coproduct decomposition into indecomposables, and are called familial distributive categories. Surprisingly, every familial distributive category is already extensive.

The fibration functor, which counts components, always preserves coproducts. However, it is not necessarily distributive, as it may not preserve products. Indeed, it preserves products only if products of indecomposables are indecomposable. In other words, the category is the coproduct completion of a cartesian category.

The category of G-Set $_{f}$ is a good example of a familial distributive category. The product of two indecomposable $G$-sets is not indecomposable. Indeed, it is well known that the Burnside rig, which describes the behavior of isomorphism classes of $G$-sets under the multiplication given by the product, provides useful information concerning the structure of the group.

\subsection{Indecomposable objects}

An object $A$ is indecomposable in a category if, whenever $(Z, x, y)$ is a coproduct, any $f: A \longrightarrow Z$ factors through either $x$ or $y$, but not both. If the category has coproducts, 


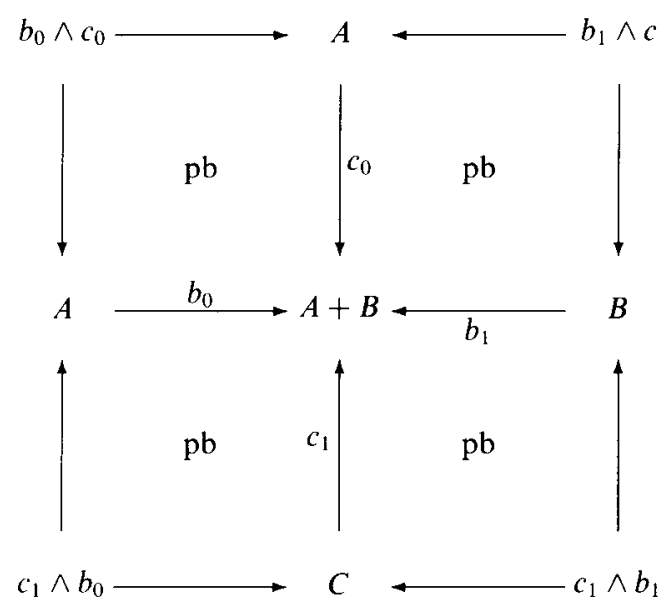

Fig. 24 .

this means, in particular, that indecomposables are not preinitial. In general, we have the following characterization.

Proposition 5.1. $A$ is indecomposable if and only if $\mathbf{X}(A,-): \mathbf{X} \longrightarrow$ Set preserves coproducts.

If the category is extensive, there are a number of other characterizations of indecomposables. The following result is well known (Diers 1985).

Proposition 5.2. In an extensive distributive category, the following are equivalent:

(i) $A$ is indecomposable,

(ii) $\operatorname{Sub}_{\mathrm{c}}(A)=\mathbf{2}$,

(iii) any map $f: A \longrightarrow 1+1$ factors through $\top$ or $\perp$, but not both,

(iv) whenever $A \simeq X+Y$, either $X$ or $Y$ is 0 , but not both,

(v) $\mathbf{X}(A,-): \mathbf{X} \longrightarrow$ Set is a distributive functor.

The following analogue of the Krull-Schmidt lemma for modules of a ring is true.

Lemma 5.3. In an extensive distributive category, if $A$ is indecomposable and $\left\langle c_{0} \mid c_{1}\right\rangle$ : $A+C \stackrel{\cong}{\longrightarrow} A+B$, then $B \simeq C$.

This can be obtained by examining the diagram in Figure 24. Each row and column is a coproduct. Either $b_{0} \wedge c_{0} \simeq A$ or $c_{1} \wedge b_{0} \simeq b_{1} \wedge c_{0} \simeq A$. In either case, $B \simeq C$.

An indecomposable (coproduct) decomposition of an object $X$ is given by a pair $\left(\left[A_{1}, \ldots, A_{n}\right], \alpha\right)$, consisting of a list of indecomposable objects together with an isomorphism $\alpha: A_{1}+\ldots+A_{n} \longrightarrow X$ given by the detachments $\alpha_{i}: A_{i} \longrightarrow X$. As is the case for modules, we can now prove the following corollary.

Corollary 5.4. In an extensive distributive category, if $\left(\left[A_{1}, \ldots, A_{n}\right], \alpha\right)$ and $\left(\left[B_{1}, \ldots, B_{m}\right], \beta\right)$ are indecompsable decompositions of $X$, then $n=m$ and there is a permutation $\sigma$ and isomorphisms $\rho_{i}: A_{i} \longrightarrow B_{\sigma(i)}$ such that $\alpha_{i}=\rho_{i} \cdot \beta_{\sigma(i)}$. 


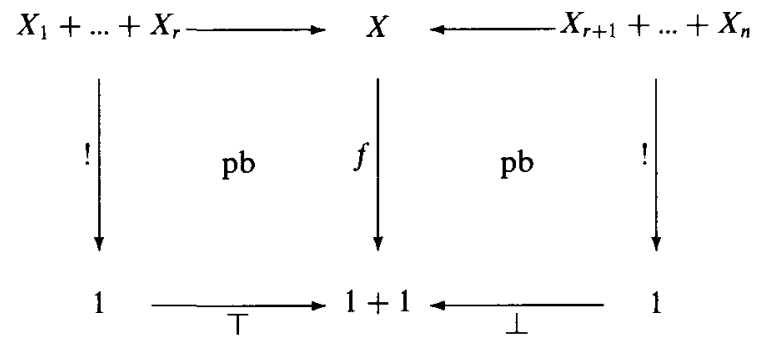

Fig. 25.

Thus, any two decompositions to indecomposables in an extensive distributive category are equivalent.

If $\mathbf{X}$ is a distributive category with an initial object in which every object has a finite indecomposable decomposition (including the empty decomposition), we shall call it a familial distributive category. In particular, note that the decomposition of 0 is ([], $\left.0_{0}\right)$, and there can be no other preinitials non-isomorphic 0 , as they would have the same decomposition, and so would be isomorphic. Thus, a familial distributive category is certainly solid.

Proposition 5.5. Familial distributive categories are extensive.

To prove this, it suffices to prove that $(1+1, T, \perp)$ is splitting. However, any $f$ : $X \longrightarrow 1+1$ can be decomposed as $\left\langle f_{1}|\ldots| f_{n}\right\rangle: X_{1}+\ldots+X_{n} \longrightarrow 1+1$, where each $X_{i}$ is indecomposable. This means that each $f_{i}: X_{i} \longrightarrow 1+1$ factors through either $T$ or $\perp$. Suppose $X_{1}, \ldots, X_{r}$ factor through $T$, and $X_{r+1}, \ldots, X_{n}$ factor through $\perp$, then I claim the diagram in Figure 25 holds. Certainly each square commutes. Considering any map $h: Z \longrightarrow X$, it is determined by the maps from its indecomposables in its decomposition $h_{i}: Z \longrightarrow X$. However, each such map must factor through one indecomposable component of $X$. If $h . f=!$. T, each component must factor through one of $X_{1}, \ldots, X_{r}$, and thus the left square is a pullback. Similarly, the right square is a pullback.

\subsection{The family construction}

Let $\mathscr{S}: \mathbf{D}_{0} \longrightarrow$ Set denote the initial functor of distributive categories with an initial object to Set. Thus, $\mathscr{S}$ gives an equivalence to $\mathbf{S e t}_{f}$. We may take $\mathbf{D}_{0}$ as also being initial in the category of elementary distributive categories with functors that preserve designated coproducts so that the objects of $\mathbf{D}_{0}$ are coproduct "shapes" of the form $(1+1)+(1+(1+1))$.

Given any category $\mathbf{X}$, the internal categories of $\mathbf{X}$ form a 2-category cat(X). In particular, the objects of $\mathbf{X}$ form discrete categories, so that, given any internal category $\mathscr{C}$, one may form the category whose objects are functors from discrete categories to $\mathscr{C}$, and whose maps are pairs $(t, \alpha): f \longrightarrow g$, where $t$ is a functor between the discrete categories (i.e. a map between the objects) and $\alpha: f \Rightarrow t . g$ is a natural transformation. This category is denoted $\mathbf{X} / / \mathscr{C}$, and is called the double slice category over $\mathscr{C}$. It is an 


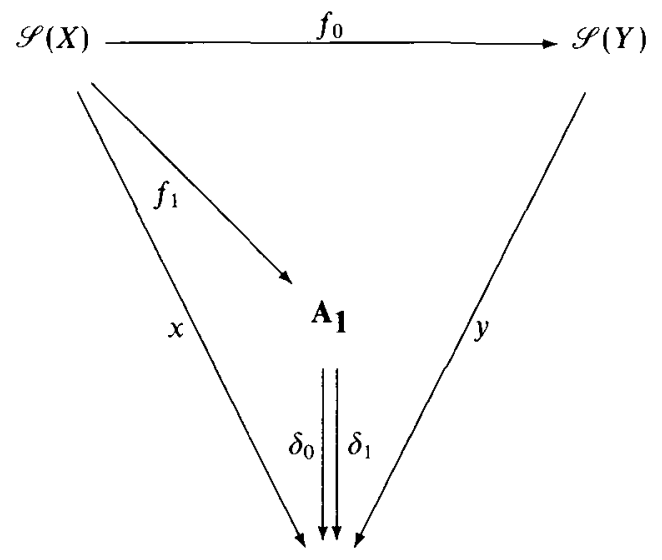

$\mathbf{A}_{0}$

Fig. 26.

example of a "super comma category" (Mac Lane 1971), and it gives rise to a fibration over X (Schumacher and Street 1988).

We may further refine this by considering any functor $F: \mathbf{Y} \longrightarrow \mathbf{X}$. Then, by $F / / \mathscr{C}$ is meant the category whose discrete objects are in $\mathbf{Y}$ but whose natural transformations are given in the obvious manner in $\mathbf{X}$.

If $\mathbf{A}$ is a small category, it is an internal category in Set, so we may form $\mathscr{S} / / \mathbf{A}$. Explicitly, the objects of this category are maps $x: \mathscr{S}(X) \longrightarrow \mathbf{A}_{0}$, while the maps are given by pairs $\left(f_{0}, f_{1}\right)$, where $f_{0}: X \longrightarrow Y$ and $f_{1}: \mathscr{X} \longrightarrow \mathbf{A}_{1}$ such that the diagram in Figure 26 holds, $x=f_{1} . \delta_{0}$ and $\mathscr{P}\left(f_{0}\right) \cdot y=f_{1} . \delta_{1}$.

This category is also sometimes denoted Fam(A), and is called the category of families of A. Notice that each object of $\mathscr{S} / / \mathbf{A}$ may be regarded as consisting of a shape, together with a labeling of the leaves of that shape by the objects of $\mathbf{A}$. As such, there is a natural extension of any functor of $\mathbf{A}$ into a category with designated coproducts that takes these labeled shapes to the designated coproducts, and the maps to the obvious coproduct comparison maps. This gives the following proposition.

Proposition 5.6. $\mathscr{S} / / \mathbf{A}$ is the free coproduct completion of $\mathbf{A}$.

A category $\mathbf{A}$ is said to have familial finite products if for every $A_{1}, \ldots, A_{n}$, the category of discrete cones $\mathbf{A} /\left(A_{1}, \ldots, A_{n}\right)$ has finitely many connected components and a final object in each component. I shall call each such final object a familial product of $A_{1}, \ldots, A_{n}$ in $\mathbf{A}$. In particular, the components of $\mathbf{A} /()$ are the components of $\mathbf{A}$, each of which must have a final object, each of which I shall call a familial final object of $\mathbf{A}$.

A has familial finite products if and only if $\mathscr{S} / / \mathbf{A}$ has actual finite products. It can be seen that this product distributes over the coproduct in two ways. First, the coproduct completion is a full faithful subcategory of $\mathbf{S e t}^{\mathbf{A}^{\text {op }}}$ and the products are preserved in this embedding, so they certainly distribute over the coproduct. Second, notice that the familial products of $\mathbf{A}$ give a functor $-\times-: \mathbf{A} \times \mathbf{A} \longrightarrow \mathscr{S} / / \mathbf{A}$, which can be extended "bilinearly" to the coproduct completion in each coordinate. This functor 
$(-\times-)^{*}: \mathscr{S} / / \mathbf{A} \times \mathscr{S} / / \mathbf{A} \longrightarrow \mathscr{S} / / \mathbf{A}$ gives the products in $\mathscr{S} / / \mathbf{A}$, and, by construction, it preserves coproducts in each coordinate, showing that $\mathscr{S} / / A$ is distributive.

Proposition 5.7. $\mathscr{S} / / \mathbf{A}$ is a distributive category if and only if $\mathbf{A}$ has familial finite products.

There is an obvious coproduct preserving functor $\delta: \mathscr{S} / / \mathbf{A} \longrightarrow \mathscr{S}$ that simply forgets the structure given by $\mathbf{A}$. This allows one to regard the situation as a fibration (Schumacher and Street 1988). In particular, the category $\mathbf{A}$ can be recaptured as the fibre over 1 . This also allows the observation that each object of $\mathbf{A}$ becomes indecomposable in $\mathscr{S} / / \mathbf{A}$, as 1 is indecomposable in $\mathbf{D}_{0}$ and $\operatorname{Ind}(\mathscr{S} / / \mathbf{A})$ (the full subcategory of indecomposables in $\mathscr{S} / / \mathbf{A}$ ) is simply A. Furthermore, each object in $\mathscr{S} / / \mathbf{A}$ has, by definition, a finite indecomposable decomposition, which leads to the following corollary.

Corollary 5.8. If $\mathbf{A}$ has familial products, then $\mathscr{S} / / \mathbf{A}$ is extensive distributive.

If $\mathbf{A}$ is cartesian, so that it has finite products, then certainly it has familial products. Furthermore, any functor $H: \mathbf{A} \longrightarrow \mathbf{X}$ of $\mathbf{A}$ to a distributive category $\mathbf{X}$ having an initial object that preserves products has its extension $H^{+}: \mathscr{S} / / \mathbf{A} \longrightarrow \mathbf{X}$ a functor that preserves coproducts. This gives the following corollary.

Corollary 5.9. If $\mathbf{A}$ is cartesian, the free distributive completion is $\mathscr{S} / / \mathbf{A}^{*}$.

Furthermore, observe that in this case products of indecomposables are indecomposable, so that we have the following corollary.

Corollary 5.10. $A$ is cartesian if and only if

(i) products of indecomposables are indecomposable in $\mathscr{S} / / \mathbf{A}$,

(ii) $\delta: \mathscr{S} / / \mathbf{A} \longrightarrow \mathbf{S}$ is distributive.

While $\delta$ is distributive for cartesian $\mathbf{A}$, it will not in general preserve all finite limits. For example, we may take $\mathbf{A}$ to be an algebraic theory with two distinct elements $1 \stackrel{0}{\longrightarrow} A$ (e.g. the theory of rings). In $\mathscr{S} / / \mathbf{A}$ the map $\langle 0 \mid 1\rangle: 1+1 \longrightarrow A$ is monic. It is easily seen that $\delta$ does not preserve this monic by counting components. This should be a sharp reminder that distributive functors between extensive categories need not behave well on arbitrary limits.

So far we have seen that every $\mathscr{S} / / \mathbf{A}$, where A has familial finite products, is familial distributive. In fact, the converse is also true (to equivalence).

Theorem 5.11. $\mathbf{X}$ is familial distributive if and only if $\mathbf{X} \simeq \mathscr{P} / / \mathbf{A}$, where $\mathbf{A}$ has familial finite products. Furthermore, $\mathbf{A} \simeq \operatorname{Ind}(\mathbf{X})$.

Clearly, as each object has an indecomposable decomposition, it can be regarded as a family of indecomposable objects. Furthermore, the maps between objects can also be represented familially, and this shows $\mathscr{S} / / \operatorname{Ind}(\mathbf{X}) \simeq \mathbf{X}$. Any equivalence of categories will carry indecomposables onto indecomposables, so the $\mathbf{A}$ of the theorem is always equivalent to $\operatorname{Ind}(\mathbf{X})$.

This means that, in discussing familial distributive categories, one can equivalently deal with categories with familial finite products. This alternative view of familial distributive categories is sometimes fruitful. 


\subsection{Object decidability}

$\mathscr{S} / / \mathbf{A}$ is distributive with finite limits, and so is Schanuel/Lawvere distributive if and only if $\mathbf{A}$ has familial finite limits. Alternatively, $\mathbf{X}$ is a finitely complete familial distributive category if and only if $\operatorname{Ind}(\mathbf{X})$ has familial finite limits. We have noted that one way in which a Schanuel/Lawvere distributive category arises naturally from an extensive distributive category is when every object is decidable. Familial distributive categories in which every object is decidable have a number of interesting properties.

Notice that equalizers are detachable when every object is decidable. This means that maps to indecomposables must either be epic or initial. Similarly, considering the equalizer of two maps from an indecomposable: either they are equal everywhere or nowhere. A rather striking observation is that graphs of maps are detachable, giving the following lemma.

Lemma 5.12. In an internally decidable familial distributive category there are only finitely many maps between any two objects.

In particular, considering an endomorphism $f: A \longrightarrow A$ of an indecomposable object $A$, because there are only finitely many endomorphisms, it follows that $f^{n}=f^{m}$ for some $n \neq m$. Using the fact that $f$ is epic, we obtain $f^{n-m}=i$, so $f$ is an isomorphism. This provides an analogue of Schur's lemma.

Proposition 5.13. In internally decidable familial categories, all endomorphisms of indecomposables are isomorphisms.

This has the following rather surprising consequence.

Corollary 5.14. A category A with familial products has all objects $\mathscr{S} / / \mathbf{A}$ decidable if and only if $\mathbf{A}$ has no non-trivial idempotents.

If $\mathscr{S} / / \mathbf{A}$ is internally decidable, every idempotent of an indecomposable must be an isomorphism, whence the identity. Conversely, consider the component of the product into which the diagonal map embeds: the composition of a projection and the diagonal map provide an idempotent on that component. If it is trivial, this component must be the diagonal, giving decidability of the object.

\subsection{The Burnside rig}

The isomorphism classes of objects of any elementary distributive category under the sum and product form a (commutative) rig which is called the Burnside rig. ${ }^{\dagger} \mathrm{A}$ rig is a quintuple, $R=\left(R_{0}, 0,1,+, \times\right)$, where $R_{0}$ is the underlying object, 0 and 1 are constants and identities for + and $\times$, which are associative binary operations with + commutative and $x$ distributing over + . A rig is commutative if $x$ is commutative, and cancellative if $x+z=y+z \Rightarrow x=y$. Notice that in a cancellative rig $a \times 0=0$, as $0+a \times 0=a \times 0+a \times 0$.

There is a reflection of rigs into rings, which uses the following construction.

\footnotetext{
$\dagger$ This name is due to Steve Schanuel and Bill Lawvere: it is a ring without negatives.
} 
Let $R$ be a rig and define the following rig structure on $\mathscr{R}(R)=R \times R / \sim$ :

$$
\begin{gathered}
(x, y)+\left(x^{\prime}, y^{\prime}\right)=\left(x+x^{\prime}, y+y^{\prime}\right) \\
(x, y) \times\left(x^{\prime}, y^{\prime}\right)=\left(x \times x^{\prime}+y \times y^{\prime}, x \times y^{\prime}+y \times x^{\prime}\right),
\end{gathered}
$$

with equivalence relation given by

$$
(x, y) \sim\left(x_{1}, y_{1}\right) \Leftrightarrow x+y_{1}+z=y+x_{1}+z,
$$

for some $z$. Clearly $\mathscr{R}(R)$ is a ring and gives the reflection of rigs to rings. It is immediate from this construction that cancellative rigs can be embedded faithfully into their enveloping ring.

It is of some interest to uncover conditions under which the Burnside rig of a distributive category is cancellative. Clearly we must have $X+A \simeq Y+A \Rightarrow X \simeq Y$. However, notice that this is a version of the Krull-Schmidt lemma, and is clearly satisfied by any $A$ that is a finite coproduct of indecomposables. An immediate observation is the following proposition.

Proposition 5.15. The Burnside rig of a familial distributive category is cancellative, and so embeds faithfully into the Burnside ring.

The Burnside rig of a familial distributive category is the free additive semi-group generated by indecomposables with multiplication given by equations of the form

$$
a \times a^{\prime}=a_{1}+\ldots+a_{n}
$$

derived directly from the familial products.

\section{Acknowledgements}

The original form of this document was written while I was a research fellow at Macquarie University being supported by the Australian Research Council. I particularly wish to acknowledge the influence and encouragement of Ross Street throughout my stay in Sydney. Contributions to this work were made by all the participants of the various Sydney category theory seminars, who included: Mike Johnson, Max Kelly, Gordon Monro, Ross Street, Michel Thiebaud, and Bob Walters.

\section{References}

Barr, M. and Wells, C. (1985) Toposes, triples and theories. Grundlehren der mathematischen Wissenschaften 278, Springer-Verlag, Berlin, Heidelberg, New York.

Cockett, J. R. B. (1989) Distributive logic, University of Tennessee, Department of Computer Science, Technical Report CS-89-01.

Cockett, J. R. B. (1991) Conditional control is not quite categorical control. In: Birtwistle, G. (ed.) IV Higher Order Workshop, Banff 1990, Workshops in Computing 190-217.

Diers, Y. (1985) Categories of boolean sheaves of simple algebras. Springer-Verlag Lecture Notes in Mathematics 1187.

Dress, A. W. M. (1973) Contributions to the theory of induced representations. In: Algebraic K-Theory II. Springer-Verlag Lecture Notes in Mathematics 342 183-240. 
Gabriel, P. and Zisman, M. (1967) Calculus of fractions and homotopy theory. Ergebnisse der Mathematik und Ihre Grenzgebiete, New Series, 35, Springer-Verlag, Berlin, Heidelberg, New York.

Grothendiek, A. and Verdier, J. L. (1972) Théorie des topos, (SGA 4, exposé I-IV). Second edition. Springer-Verlag Lecture Notes in Mathematics 269-270.

Johnstone, P. T. (1977) Topos theory, Academic Press, London, New York, San Francisco.

Lintner, H. (1976) A remark on Mackey-functors. Manuscripta math. 18 275-278.

Mac Lane, S. (1971) Categories for the working mathematician, Springer-Verlag, Berlin, Heidelberg, New York.

Schumacher, D. and Street, R. H. (1988) Some Parameterized Categorical Concepts. Communications in Algebra 16 (11) 2313-2347.

Sydney Category Seminars (1988) Sydney category seminar abstracts.

Sydney Category Seminars (1990) Sydney category seminar abstracts. 